\title{
Focal Neurological Deficit Secondary to Severe Hyponatraemia Mimicking Stroke
}

\author{
Ahmad Nawid Latifi ${ }^{1}$, Vanitha Gopal ${ }^{1}$, Sina Raissi ${ }^{2}$ \\ ${ }^{1}$ Department of Internal Medicine, Saint Mary's Hospital, Waterbury, CT, USA \\ ${ }^{2}$ Department of Nephrology, Saint Mary's Hospital, Waterbury, CT, USA
}

Received: 29/07/2019

Accepted: $17 / 09 / 2019$

Published: 11/10/2019

How to cite this article: Latifi AN, Gopal V, Raissi S. Focal neurological deficit secondary to severe hyponatraemia mimicking stroke. EJCRIM 2019;6: doi:10.12890/2019_001244.

Conflicts of Interests: The Authors declare that there are no competing interest

This article is licensed under a Commons Attribution Non-Commercial 4.0 License

\section{ABSTRACT}

Hyponatraemia is a common electrolyte abnormality seen by internists. Clinical features of hyponatraemia are primarily related to CNS dysfunction, and depend on the severity and acuity of changes in serum sodium concentration. Neurological manifestations of hyponatraemia range from nausea and malaise, with a mild reduction in the serum sodium, to lethargy, decreased level of consciousness, headache, seizures and coma in extreme cases. Focal neurological deficits are very rare in the setting of hyponatraemia. Here, we describe a patient with acute severe symptomatic hyponatraemia presenting with focal neurological deficits that resolved after correction of acute hyponatraemia.

\section{LEARNING POINTS}

- A rare presentation of hyponatraemia is described.

- Neuroimaging should be performed in patients with focal neurological deficits and hyponatraemia in order to rule out other serious neurological diseases.

- Correction of severe hyponatraemia can result in resolution of focal neurological deficits.

\section{KEYWORDS}

Hyponatraemia, focal neurological deficit, seizure

\section{CASE PRESENTATION}

A 56-year-old woman with a medical history of hypertension, hypothyroidism and chronic alcoholism was brought to the emergency department for evaluation of confusion, slurred speech and left-sided weakness that began 1 hour before arrival. On physical examination, the patient was afebrile with moist mucous membranes and blood pressure of 145/80 mm Hg. Her heart rate was 65 and she was saturating $97 \%$ on room air. Her neurological examination was remarkable for left-sided facial droop and 3/5 weakness of the left upper extremity with left-sided neglect. She had a score of 4 on the National Institute of Health Stroke Scale and a score of 2 on the Recognition of Stroke in the Emergency Room (ROSIER) scale.

During the initial evaluation in the emergency department before head computed tomography (CT) could be completed, the patient developed status epilepticus and required intubation. She was loaded with levetiracetam and placed on seizure precautions. Subsequently, a head CT was negative for intracranial haemorrhage and tissue plasminogen activator (tPA) was commenced. Laboratory work-up revealed a serum sodium of $109 \mathrm{mEq} / \mathrm{l}$. The patient was transferred to the intensive care unit for management of severe acute symptomatic hyponatraemia. The tPA infusion was stopped and she was given hypertonic (3\%) sodium chloride with an appropriate rise in serum sodium to $115 \mathrm{mEq} / \mathrm{l}$ within the first few hours. Magnetic resonance imaging (MRI) of the brain was negative for acute stroke. Further laboratory investigations revealed a normal serum thyroid-stimulating hormone, lipid profile and cortisol levels. She also had normal serum creatinine and glucose 
levels. The patient's serum osmolality was 238 mOsm/I (275-295 mOsm/l) and her urine osmolality was 27 mOsm/l (50-1200 mOsm/l). Based on these investigations, the patient was thought to have psychogenic polydipsia or beer potomania. Further history obtained from her husband revealed that she consumes 2-3 litres of water per day as well as at least one bottle of wine per day. Additionally, the patient was not on any chronic medications. Over the next 2 days, the patient was placed on fluid restriction and received intermittent doses of desmopressin and hypotonic fluids to avoid rapid correction. Her facial droop and left-sided weakness improved by the time she was weaned from sedation and she was extubated. She had no recurrent episodes of seizure. Prior to discharge, the patient's serum sodium was $133 \mathrm{mEq} / \mathrm{l}$ and she was sent home with outpatient follow-up.

\section{DISCUSSION}

Hyponatraemia is defined as a serum sodium concentration below $135 \mathrm{mEq} / \mathrm{l}$, which reflects a relative excess of total body water (TBW) in relation to sodium content. Hyponatraemia with low urine osmolality indicates suppressed antidiuretic hormone (ADH)and is due to either excessive fluid intake (psychogenic polydipsia) or very low osmolar load (beer potomania or malnutrition). Hyponatraemia with elevated urine osmolality indicates elevated $\mathrm{ADH}$ and is divided to three categories based on total body volume: (1) hypovolemic hyponatraemia, (2) euvolemic hyponatraemia and (3) hypervolemic hyponatraemia based on TBW and total body sodium content. Hyponatraemia which develops in a period of less than 48 hours is considered acute. Chronic hyponatraemia is defined as hyponatraemia that develops over more than 48 hours. Hyponatraemia-induced cerebral oedema occurs most of the time with rapid reductions in the serum sodium concentration, usually in less than 24 hours. It most often occurs in patients with self-induced water intoxication due to primary polydipsia, exerciseassociated hyponatraemia or in postoperative patients given large quantities of hypotonic fluid.

The presence of symptoms and signs of hyponatraemia is dependent on the severity and acuity of changes in serum sodium concentrations. Symptoms and signs are primarily related to CNS dysfunction. Of note, patients may also have clinical manifestations related to concomitant volume depletion and/or underlying diseases that predispose the patient to the electrolyte derangements. The mechanism of neurological dysfunction in the setting of hyponatraemia is the presence of an osmolar gradient created by the low serum osmolality, resulting in intracerebral osmotic fluid shifts and brain oedema. This primarily occurs in acute hyponatraemia due to rapid changes in serum sodium concentrations. In chronic hyponatraemia, the brain adapts itself to hyponatraemia through the generation of idiogenic osmoles. This is a protective mechanism that reduces the degree of cerebral oedema. The generation of idiogenic molecules begins on the first day hyponatraemia is present and is completed within several days. Hence, chronic hyponatraemia patients may appear asymptomatic ${ }^{[1]}$.

The neurological manifestations of hyponatraemia range from nausea and malaise, with a mild reduction in the serum sodium, to lethargy, decreased level of consciousness, headache, seizures and coma in extreme cases. In severe cases, tentorial herniation with subsequent brain stem compression and respiratory arrest, resulting in death, can occur. Severe symptoms do not manifest until the sodium level is below $120 \mathrm{mmol} /\left[^{[2]}\right.$. Acute and chronic hyponatraemia cannot always be distinguished from each other based on symptomatology ${ }^{[3]}$.

Focal neurological deficits (FND) secondary to hyponatraemia are rare with an estimated occurrence rate of $5 \%$ in severe hyponatraemia ${ }^{[4]}$. The mechanism of FND secondary to hyponatraemia is not well understood. Hyponatraemia causes neurological symptoms through cellular swelling followed by brain oedema. As this cellular swelling is not limited to one focal cerebral region but affects the whole brain, it ordinarily results in generalized central nervous dysfunction. Hyponatraemia can present as a variety of FND including hemiparesis, monoparesis, rigidity, extrapyramidal type tremor, cerebellar ataxia, nystagmus and anisocoria ${ }^{[5]}$. It is also critical to remember that FND can be a sign of concurrent neurological disease including brain tumours, ischaemic stroke, intracranial haemorrhage and meningoencephalitis ${ }^{[6]}$. Therefore, a patient with FND in spite of hyponatraemia or other metabolic derangement should undergo neuroimaging to rule out other life-threatening conditions.

The treatment of hyponatraemia depends on the underlying cause and acuity of onset. Acute symptomatic hyponatraemia is usually treated with hypertonic (3\%) sodium chloride with the goal of raising the serum sodium concentration by 4-6 mEq/l in less than 6 hours during the initial phase of treatment, not to exceed a 6-8 mEq/l correction per 24 hours. During treatment, patients should be closely monitored to avoid rapid correction and overcorrection. Both situations may result in osmotic demyelination syndrome.

\section{REFERENCES}

1. Sahay M, Sahay R. Hyponatremia: a practical approach. Indian J Endocrinol Metab 2014;18(6):760-771.

2. Walmsley RN, Watkinson LR, Koay ES. Cases in chemical pathology: a diagnostic approach. 3rd ed. Singapore: World Scientific; 1992.

3. Kuthiah N, Er C. Reset osmostat: a challenging case of hyponatremia. Case Rep Med 2018;2018:5670671.

4. Nigro N, Winzeler B, Suter-Widmer I, Schuetz P, Arici B, Bally M, et al. Symptoms and characteristics of individuals with profound hyponatremia: a prospective multicenter observational study. J Am Geriatr Soc 2015;63:470-475.

5. Daggett P, Deanfield J, Moss F. Neurological aspects of hyponatraemia. Postgrad Med J 1982;58(686):737-740.

6. Bokemeyer A, Dziewas R, Wiendl H, et al. Hyponatremia upon presentation to the emergency department - the need for urgent neuroimaging studies. Sci Rep 2017;7(1):1953. 